\title{
Intelligent Networked Flight Test Instrumentation for a new Fighter Prototype
}

\author{
Guillermo Martínez Morán \\ ${ }^{1}$ Airbus Defence\&Space, Paseo John Lennon s/n (Getafe-Madrid) - Spain \\ guillermo.m.martinez@airbus.com
}

\begin{abstract}
:
Airbus Defence \& Space treasures more than 10 years of expertise in implementing fully network based flight test instrumentations (FTI). The developments accomplished during this long walk have brought to fruition very flexible and versatile FTI. Big platform programs (such as C295, A330-MRTT and $\mathrm{A} 400 \mathrm{M})$ have been taking good advantage from these features.

Transferring these features to a smaller aircraft (such as a fighter) has been the last challenge achieved. Restrictions due to the lack of space and crew onboard had to be overcome by implementing some intelligence onboard. This paper contains description and capabilities of the networked intelligent FTI architecture implemented in a new flight test fighter.
\end{abstract}

Key words: Intelligent, Network, FTI Architecture, Flexible, Versatile.

\section{Introduction}

Flight Test Instrumentations (FTI) architectures based in Pulse Coded Modulation (PCM), and used for decades, have been quite useful in the past and sure will remain with a gold ribbon in the memories of the FTI history.

Nevertheless, the fixed and static nature of the PCM schema derives in rigid and complex architectures, which present many implementation problems in the present context of bigger and bigger FTI installations with more and more data acquired.

This complexity is highly reduced by applying a more flexible schema like the network concept using data packets. Architecture schemas based on Ethernet networks have been widely adopted worldwide in the last 10 years in instrument aircrafts.

Nevertheless, at least in Europe, it has not been applied to fighter instrumentations, maybe due to the fact that data amount is less than the one generated in big aircrafts. However, better features and less cost are possible in fighter installations if the flexibility of the networked schema is used.

This paper presents the new FTI networked architecture for a fighter and compares it to the one used previously.

The presented architecture takes advantage of the flexibility, in order to introduce some intelligent capabilities by adding a basic computing device.
First a brief history of the journey from PCM to Ethernet Networked architectures inside Airbus Defence \& Space will be shown.

After that, main FTI systems will be described and compared both for the old and the new architecture. Systems specifically analyzed are: Data Acquisition, Data Distribution, Video, Telemetry, Recording, Time Distribution and Control \& Monitoring.

Finally, a detailed description of the intelligent device and its main features will be addressed.

\section{Brief FTI Architecture History}

This section contains the description of the evolution of FTI architectures inside Airbus Defence \& Space.

Before 2000 all FTI installations used the PCM paradigm to time stamp data acquired.Main advanced related to past installations, where the acquisition system was centralized, was the use of a distributed acquisition system.

This feature allowed to reduce the cabling needed, as all the parameters in one area were grouped in a remote acquisition unit, which was connected to the rest of the system by only two cables (time and data). Previously, each measurement implied a cable being routed from the measuring point to the acquisition system.

Nevertheless, this distributed system used a daisy chain schema, where a master unit must receive all the data coming from the slave units. These slaves were connected following a chain, 
with the poor failure resistance provided by this architecture (when a unit fails, all the units behind fails). Additionally, a bottleneck occurs in the data bus connecting all the units, as data are carried to the master unit in a cascade schema.

With the A310-BOOM Flight Test Bed program, in 2002, the new packets paradigm was applied for the first time. However, a parallel installation based on PCM and packets was put in place, in order to validate the new paradigm. For this reason the daisy chain architecture was still used and not all the flexibility and advantages from a real networked architecture were used.

The full networked architecture was for the first time applied in 2005 with the A330-MRTT prototype. After the experience gained with the A310-BOOM program, a fully networked FTI architecture was put in placed with satisfactory results. Nevertheless, available technology at that time did not allow acquiring all the information running through the aircraft buses. It was mandatory to tell the acquisition system which parameters had to be acquired. As a result, managing buses definitions was still highly critical.

With the time the technology evolved and fullacquisition capabilities for buses were introduced in the acquisition systems, reducing the complexity of programing the acquisition system.

Currently, all the new instrumented aircrafts inside Airbus Defence \& Space are fully networked and those installed in big aircrafts implements several automatizations and intelligent algorithms running on computers.

The last big step has been done in 2017 with the ultimate test fighter, which includes a fully networked architecture, with full-acquisition capability and intelligence on-board.

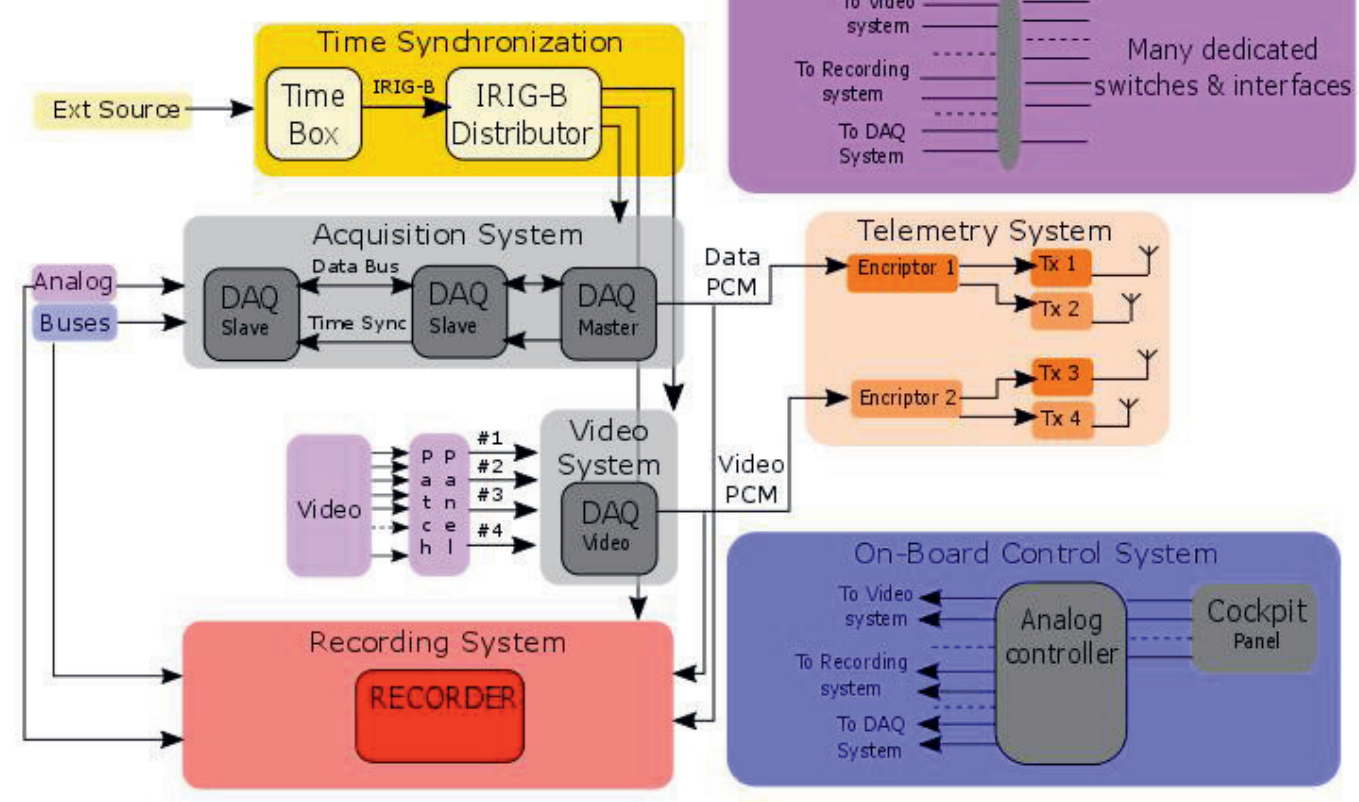

Fig.1. Legacy Top Level Architecture

\section{Data Acquisition System (DAQ)}

In the previous architecture the data acquisition system was based in following main characteristics: PCM output, daisy chain, dedicated time synchronization protocol and parsing acquisition schema.

It was a distributed system with several Remote Acquisition Units (RDAU) acquiring data all around the aircraft. Nevertheless, all acquired data were collected in a master unit which was in charge of building the PCM output with the acquired information. This master unit was connected with the RDAUs through a dedicated data bus using a serial daisy chain schema (units connected one after another). Main drawbacks of this schema are: poor failure resistance (if one unit fails all the units after it fails), high programming time, no modular programming, limited throughput between RDAUs, complexity to allocate slots for all acquired data in the PCM output.

On the contrary, the new architecture is also a distributed system but based on a star schema, where each RDAU is master of its own acquired data. These data are packetized into an IENA stream, which is sent through Ethernet link to the rest of the system. Main advantages of the 
star schema are: better failure resistance (each RDAU operates independently), no bottlenecks to pass data to other RDAUs, less allocation complexity in output stream, a more scalable system (it is easier to add a new RDAU) and in general more flexibility and data throughput.

Additionally, time synchronization of all the RDAUs was performed by using a dedicated time signal implemented in a dedicated bus (also daisy chain based). In the new architecture time synchronization among the RDAUs use a protocol over the data bus (no need for two cables), which is the same protocol of the rest of the FTI system.

Finally, the legacy schema for aircraft buses acquisition was a parsing schema, where certain known data in the buses are extracted and placed into the output stream. This schema implies a perfect knowledge of the aircraft buses definition (ICDs - Interface Control
Document), which is not always simple. Any failure in the ICDs implies a lost of the data, as the data are not acquired in origin. In contrast, the full-acquisition schema of the new architecture acquires all the data from aircraft buses. As soon as any data appears in the bus, is acquired, packetized and sent. Therefore, all data in the bus are acquired, no matter ICD is right or wrong. In case ICD was not correct, data can be interpreted in postprocessing.

Nevertheless, as the amount of acquired data excesses by far the telemetry data throughput available, a parsing acquisition schema is also programed in order to send data through the telemetry system. This stream will suffer any ICD issue, but full-acquired recorded data will be safe.

Therefore, DAQ outputs two different data streams: a full-acquisition stream and a filtered (parsed) stream.

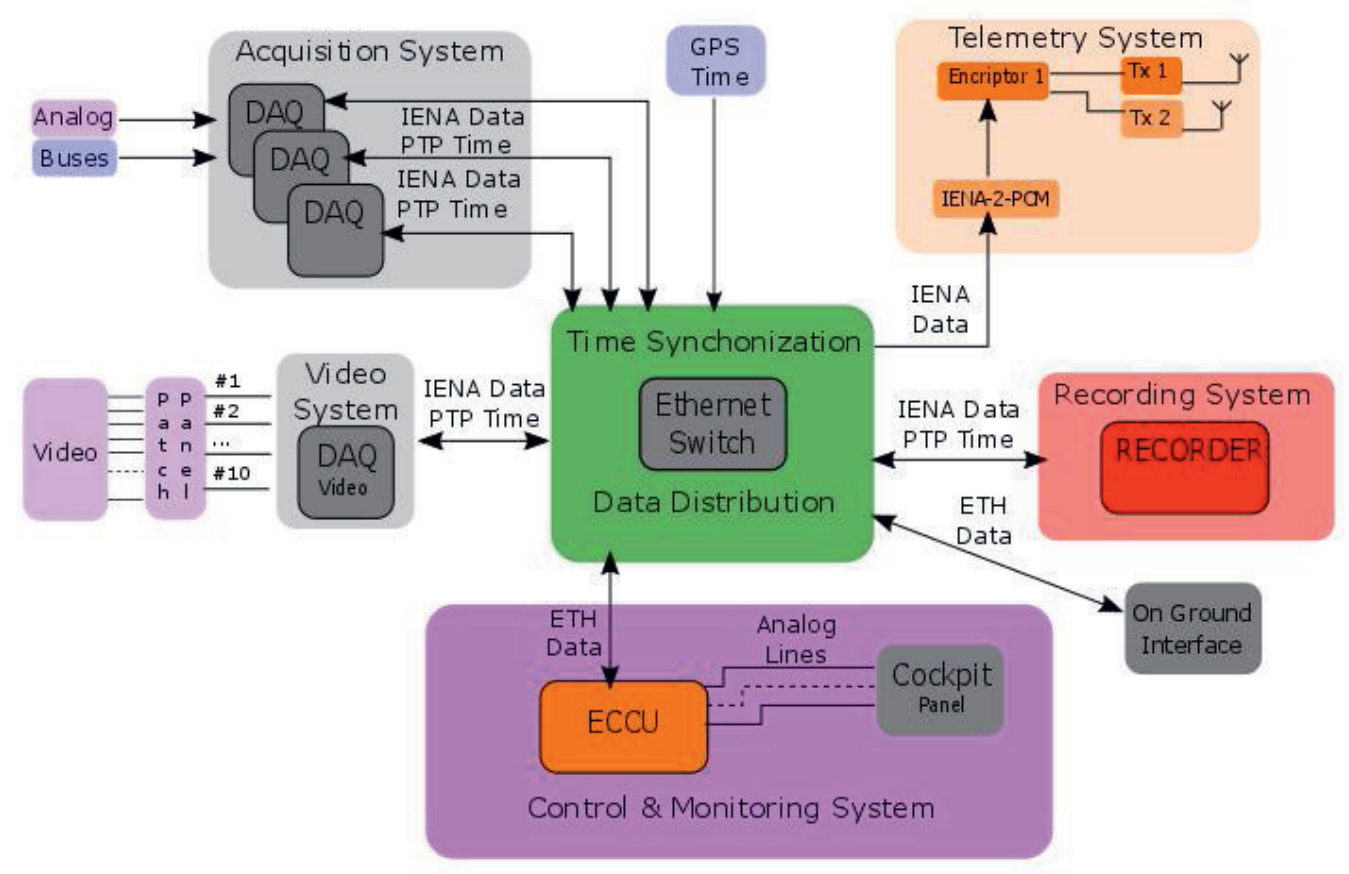

Fig. 2. New Top Level Architecture

\section{Data Distribution System}

Legacy architecture data distribution system was based on a point to point connection schema using physical wires connecting each device to another one. The devices could "speak" only with the devices connected to them by a wire.

On the other hand, the new architecture is based in a network schema, where all the devices are physically connected to a central point (Switch) through an Ethernet wire.
This network provides point-to-point communication capability, as each device in the network has a unique address. Each device can speak with any other device in the network, incrementing the working possibilities of the system. This feature is used by the Enhanced Cockpit Control Unit (ECCU) to monitor and control the FTI system.

Additionally, it is possible to send data from one source to many sinks using the multicast capability. It is possible to program in the switch where each data stream must sink. This feature 
is widely used in the architecture, as registered in Error! Reference source not found..

Moreover, this configuration can be easily changed (even on-the-fly) by configuring the switch accordingly. This feature is used to manage the 10 video data streams, selecting in each flight phase the most appropriate video stream to be shown on ground.

The data distribution system also allows distributing a time synchronization signal based on Ethernet packets to all the connected devices.

Therefore, only one cable per device provides: point-2-ponit communication with all the system devices, several data streams management and time distribution.

As there are many configuration possibilities is difficult to estimate a maximum throughput the system can handle. Nevertheless, a realistic estimation is up to $100 \mathrm{Mbps}$ per device.

Tab. 1: New Architecture Data Streams

\begin{tabular}{|c|c|c|}
\hline Type & Source & Sink \\
\hline Full-Acquisition & - DAQ & $\begin{array}{l}\text { - REC } \\
\text { - ECCU } \\
\text { - On-Ground } \\
\text { Interface }\end{array}$ \\
\hline $\begin{array}{l}\text { Telemetry } \\
\text { Acquisition }\end{array}$ & - DAQ & - REC (forensic \\
\hline $\begin{array}{c}\text { System Status } \\
\qquad 1\end{array}$ & - ECCU & - IENA-2-PCM \\
\hline $\begin{array}{c}\text { System Status } \\
\# 2\end{array}$ & - DAQ & - ECCU \\
\hline Video \#1 & \multirow{4}{*}{$\begin{array}{c}\text { - Video } \\
\text { DAQ }\end{array}$} & \multirow{4}{*}{$\begin{array}{ll}\text { - REC } & \\
\text { - Some of them } \\
\text { to } \text { IENA-2- } \\
\text { PCM }\end{array}$} \\
\hline Video \#2 & & \\
\hline Video ... & & \\
\hline Video \#10 & & \\
\hline
\end{tabular}

Video System

In the legacy architecture the video system was able to acquire record and send through telemetry 4 simultaneous video signals. As the possible position cameras were more than 4 , a physical patch panel existed in order to plug a fixed camera configuration into the encoders before each test flight.

On the other hand, in the new architecture the system capability have been extended up to 10 simultaneous video encoders. Nevertheless, the physical patch panel still remains as possible position cameras are more than 10 .

10 video data streams are always sent to the data distribution system. These data streams are also IENA streams, so they are managed in the same way as the DAQ data streams.

The switch manages what to do with each video stream. Normally, all the acquired videos are sent to the recording system and a set of them are sent to the telemetry system. Videos sent to the telemetry system can be change on-the-fly, depending on the flight needs, as it is not difficult to change the switch configuration using the ECCU.

Data throughput of each video stream is estimated to be between 1 and $1.5 \mathrm{Mbps}$, so a total amount of 10-15 Mbps for the video system are used.

\section{Telemetry System}

Previous telemetry system consisted of two different telemetry chains: one for video stream and another for data streaming. This fact was imposed by the PCM encoding schema and it forced to install a large number of devices: 2 encryptors, 4 transmitters and 4 antennas. Moreover, the available bandwidth was not interchangeable between data and video, which reduces the flexibility of the system.

By using the IENA packets concept, all data streams can be merged or filtered easily and there is no need to implement two different telemetry chains. Due to this fact, a lot of savings were done both in equipment acquisition and installation costs.

As exposed in the Data Distribution System section, data acquired to be sent through telemetry are acquired in the DAQ following the parsing paradigm in order to adapt the throughput to the available telemetry bandwidth. The selection of the data stream reaching the telemetry chain is done by the switch in the Data Distribution System, but the selection of the information contained in this stream is done by the Acquisition System.

The key part of the new telemetry architecture is the equipment in charge of embedding the IENA packets into a continuous PCM stream suitable to be sent with the current telemetry paradigms. This IENA-2-PCM device follows its own algorithm to do the job and needs a counterpart device on ground, which duty is to convert PCM-2-IENA. Therefore, in the ground segment a replica of the IENA data streams is available to monitor the aircraft.

The telemetry transmitters used in the new architecture are completely digital, so they have inside a premodulation filter, in case a PCM/FM modulation schema is used.

On top of that, all configurations can be done by software: radio frequency, bandwidth and 
power, as well as the modulation schema. All the settings are performed through the ECCU, which is also in charge of monitoring the status of the transmitters.

\section{Recording System}

The recording system in the previous architecture was devoted to record all the acquired data (video and parameters) and also to provide the total recording of the buses and some high frequency analog parameters.

In the new architecture, the recording system must record all the Ethernet data streams(see Tab.1.): full-acquisition, telemetry acquisition, video and system status.

On the one hand there is no need to perform a total recording, as the DAQ assures all the information in the buses is acquired. On the other hand, the high frequency analog parameters can also been acquired by the DAQ and sent to the data distribution system via a data stream, which will not be sent to telemetry. Therefore there is no need to perform this acquisition.

In the end, the new recording system is an Ethernet network recorder recording all the IENA packets streamed through the switch.

\section{Time Distribution}

In the legacy architecture there was a time master, which was loaded with the absolute time before each flight, using a GPS connection or a time box. After this time load, the system runs in free wheel mode affected by the time shifts of the time master. This fact is not critical while the parameters used to analyze the tests come only from the aircraft. Nevertheless, as soon as parameters from on-ground or other aircraft must be analyzed, time differences become critical.

For this reason in the new architecture the system is continuously synchronized through GPS signal. Nevertheless, there is also a way to introduce time into the system in order to operate it inside hangars. The system is able to automatically manage the time source used, following a predefined priority schema. The preferred time source is the GPS; if it is not available an IRIG-B signal is used; lastly, if none of previous signals are available it will relay on the temperature compensated internal oscillator of the time server. In this new architecture, the time master is integrated inside the switch of the data distribution system.

Talking about time distribution protocol, in previous architecture, the time distribution was done using an IRIG-B signal through a point-to- point schema. This means that one dedicated cable was installed for each device.

In this new architecture the so called Precise Time Protocol (PTP), which operates over Ethernet networks has been used. First main advantage is that there is no need to add any cable as the same data cabling is used to synchronize all the devices in the system (including DAQ which also uses PTP). Moreover, PTP provides a much accurate synchronization of 100 ns, versus tenths of microseconds of the IRIG-B signal.

\section{Control System}

The previous architecture did not have a clear defined control \& monitoring system. The system was distributed in various dedicated solutions specifically developed for each need.

The onboard control part was mainly performed by using hardware switches connected to a centralized box, which produced the specific hardware signals for each one of the elements being controlled. This solution has been very useful in the past, but it has the problem of being intrinsically rigid and space consuming.

The on-ground control part was based in the same schema, conducting the control \& monitor panel to be formed by a lot of different physical interfaces and switches to interact with each one of the devices in the installation.

In the new architecture all control capabilities have been centralized in a dedicated intelligent device called Enhanced Cockpit Control Unit (ECCU). Taking advantage of the networked architecture all the control communications are performed through the same physical interface used to send the data, hence reducing cabling complexity.

Ideally, the control protocol to be used over the network would have been Simple Network Monitoring Protocol (SNMP). Regrettably, not all the devices in the system had the capability to be commanded through SNMP or not all the parameters could be configured. For this reason, we were forced to use a different network protocol in order to configure each one of the devices (i.e. Telnet or proprietary protocols).

Anyway, as the solution is based in a software programmable system is simple to change the parameters controlled or even the way each parameter is controlled.

For those systems, which still don't have a network communication, a dedicated interface has been put in place (i.e. RS-232 bus for telemetry transmitters). 
This centralized schema has allowed also substituting all the hardware switches and interfaces by only one dedicated monitoring port in the monitor panel. Through this port, thanks to a dedicated software tool, all the system can be controlled from a single point when the aircraft is on ground.

\section{Monitoring System}

As in the control system case, the monitoring system capabilities were also distributed and fully dependent on the possibilities of each device. Some of them did not even have monitoring capability (i.e. telemetry transmitters).

For example, the recording system provided limited amount of information through a dedicated RS-232 bus, which was acquired by the acquisition system and included in the PCM to be sent through telemetry. On the other hand, the acquisition system provided its own status parameters included in the PCM generated be itself.

In the new architecture, almost all the monitoring capabilities have been centralized in the ECCU. In all the cases where it has been possible to monitor the system using SNMP over the network it has been done. On the other hand, some devices were not network connected and for this reason specific interfaces have been put in place.

\section{Current Enhanced Cockpit Control Unit (ECCU) capabilities}

This section describes deeply all the functions the ECCU performs inside the new architecture.

\section{System monitoring}

The ECCU acts as a centralized monitoring system of all those devices, which does not provide a sufficient level of status report. The ECCU collect status data from those devices and packed them into IENA packets, which are send through the network both to the telemetry and recording system. The data collection schema is a polling one (systems are periodically asked about the parameters).

The telemetry transmitters are one of the devices monitored. Data collection is performed following the specific manufacturer protocol through a RS-232 bus. Parameters extracted are related to configuration control (transmitting frequency, bandwidth, modulation, etc...) and environmental conditions (working temperature).

This is the only device which does not provide a network interface. Nevertheless, thanks to the flexible design of the ECCU it is possible to combine several physical interfaces.
Another monitored system is the recorder. It is monitored using an SNMP client accessing all the relevant information. A big number of working conditions of the main unit are collected: data throughput, recording time remaining, time synchronization status, working temperature, etc... Additionally, all the information related to the input channels being recording is monitored (i.e. Ethernet throughput).

Finally, the network switch is also controlled. As in the recording system, the SNMP protocol through the network connection is used. All the parameters regarding working status (Ethernet port status and data throughput of each port) and environmental conditions (temperature) are polled.

As the switch also acts as time master for the whole system, time synchronization status is also monitored: time source used and synchronization status.

\section{System Own-Care}

The monitoring capability can be used in conjunction with the intelligence of the ECCU to take automatic care of all the parameters, which must be monitored to prevent failures in the installation.

As an example, in this aircraft, the ECCU is constantly monitoring telemetry transmitter's temperature to assure it stay below the maximum working environmental. In case, the device gets overheated automatic correction measures are taken by means of modifying the radio-frequency output power.

\section{System control and reconfiguration}

The same interfaces used to monitor transmitters, recorder and switch can be used to configure the devices. The telemetry transmitters are configured using the proprietary protocol through RS232 bus, whereas the switch and the recorder are configured using the network interface. Regrettably, the SNMP writing capability has not been implemented neither in the switch nor the recorder. Therefore other means have been used.

Configuration of the recorder has been done through network interface using TMATS commands or some proprietary commands.

On the other hand, configuration of the switch must be done using commands through a Telnet connection.

This configuration capability of the switch is used to select which videos are being transmitted through telemetry. The pilot has 
some buttons on the cockpit with which he can select the set of videos to be sent. This set can consist of any combination of the available 10 video sources. The ECCU reads the buttons positions and reconfigure, in real time, the multicast routing table of the switch, changing the videos sent to telemetry.

Moreover, other complex real-time concepts can be implemented. As an example, by combining the use of switch and recorder reconfiguration the real-time access to past measurements can be easily implemented. This concept is explained in the paper "Real-Time access to past measurements using bidirectional communication (TmNS)" included among the papers of this ETTC2018.

\section{System Intelligence}

As all the IENA packets containing information acquired by the DAQ runs through the network and are received by the ECCU, it is quite easy to read any parameter contained in those packets and program the ECCU to take an automated action, by reconfiguring any device in the system.

\section{Conclusions}

A new flight test instrumentation architecture based on a fully networked concept has been implemented in a new flight test aircraft of an existing fighter. This new concept has allowed a significant reduction in the cabling complexity and in the number of devices needed to be installed on the aircraft.

Additionally, the architecture allows having a more flexible, scalable and capable system.

In terms of capacity, the networked schema provides the possibility to handle much more data (up to $100 \mathrm{Mbps}$ per device).

Flexibility has been improved, by providing better communication possibilities among the devices being part of the system. Additionally, sending the data through IENA packets allow flexibility in the data stream impossible when using PCM schema.

On top of all, an intelligent device has been included, allowing taking advantage of the inherent flexible schema of the networked instrumentation. This intelligent device centralize all the control and monitoring tasks, which provide the capability to easily implement and change any action required in the system. As it also receives all data acquired by the FTI, it is possible to implement automated actions based on data acquired. 